\title{
Improved Unequal-Clustering and Routing Protocol
}

\author{
Mohammad Ali Alharbi and Mario Kolberg, Senior Member, IEEE
}

\begin{abstract}
Increased network lifetime is a desired property of low-powered and energyconstrained Internet of Things (IOT) devices that are deployed in wireless network environments. Clustering is used as a technique in multiple solutions to improve overall network lifetime. Further variants in the clustering process are defined to optimize the results. One such variant is equal clustering, where all the clusters have the same size. However, this approach suffers from the issue of nodes closer to the base station (BS) dying out earlier. As an alternative, unequal clustering is proposed, where clusters close to the BS are of smaller size; thus, cluster heads (CHs) consume a substantial proportion of their energy for being acting as data forwarding nodes. In this paper, we propose an unequal clustering approach with the BS at the center of a circular area. The size of each cluster is fixed and computed based on the node density of the area. The number of clusters increases from outwards to inwards towards the BS. The results show considerable performance gain over selected benchmark works.
\end{abstract}

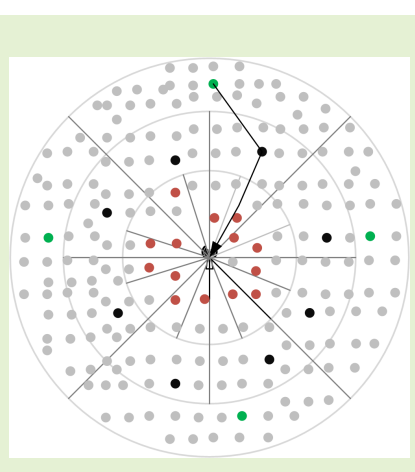

Index Terms - unequal clustering, ring clusters, fixed-area clustering

\section{INTRODUCTION}

$\mathbf{I}$ NTERNET-powered sensing devices have revolutionized different technology domains. These Internet of Things (IoT) devices are low-power wireless sensor nodes that are randomly distributed in an area to monitor various environmental and security parameters. IoT has made possible the remotely control of heterogeneous networks and is widely used in industrial applications. These are resource-constrained devices that are randomly deployed in dynamic wireless environments [1]. They are self-organizing, and transmission is performed in a multihop fashion [2]. The most critical resource of these devices is battery power that directly links to network lifetime. All device operations, such as self-organization, communication with other nodes, synchronization and transmission of routing information, are dependent on battery power [3]. Therefore, there is a need to design an energy-efficient algorithm to increase the lifetime of the network [4].

However, for short-range sensing devices deployed in a wider area, collected data cannot be sent to the sink node directly in a single-hop fashion [5]. Thus, multi-hop communication requires the development of routing protocols specified for multihop networks [6], [7]. Initially, the concept of clustering is used as a solution to overcome network-lifetime problems. In clustering, the process of Cluster-Head $(\mathrm{CH})$ selection is important to increase network lifetime for shorter area sizes. This process considers a variety of parameters such as node residual battery, degree of connectivity and mobility of nodes [7]. For larger areas, efficient routing via multiple hops in networks is also desired [6].

Clustering is a widely discussed solution for the hot-spot problem in Wireless Sensor Networks. Existing solutions have three major issues; 1) The process of clustering is dynamic in which clusters are formed irrespective of distance between $\mathrm{CH}$ and CMs. This causes higher energy consumption for lower throughput. 2) The method of equal clustering is adopted without considering that the nodes closer to BS have dual responsibilities where these nodes not only have to work as data forwarding nodes of previous $\mathrm{CHs}$ but also data collectors from their own member nodes. 3) For large area networks, the communication of $\mathrm{CH}$ and $\mathrm{BS}$ is performed in multiple hops. Existing solution considering the process of $\mathrm{CH}$ selection irrespective of routing solution. This causes hot-spot problem where network area get disconnected while still having nodes with energy.

Different types of clustering, such as equal and unequal clustering [16], are considered in different solutions. Equal clustering creates equal size clusters irrespective of distance from the BS, whereas in unequal clustering, the size of the cluster is smallest near the BS and increases as the distance of the cluster from the BS increases [16]. In equal clustering, each cluster have same number of nodes and size. During intercluster routing, the nodes in cluster near to BS are overloaded with data coming from farther node and therefore, die out quickly and cause hot-spot problem. To handle this problem, unequal clustering is proposed where the nodes closer to the BS have smaller size and lesser number of nodes, whereas as farther clusters have larger number of nodes [16].

Different existing solutions considered clustering and routing as separate issues without considering equal or unequal clustering [8]-[15]. These protocols are either based on singlehop communication with specific percentages of nodes as $\mathrm{CHs}$ [7] or on multi-hop routing [7]. Moreover, the positioning of $\mathrm{CHs}$ in the network area is random; thus, $\mathrm{CHs}$ can be 
placed inappropriately, resulting in performance degradation [10]. Clustering and routing is considered as a single unified problem in [15]. However, this solution did not solve the energy-optimization problem through unequal clustering.

The solution proposed in this work treats clustering and routing as a unified problem using unequal and fixed-areabased clusters considering position of the BS close to the center of the area. After identifying the clusters and $\mathrm{CHs}$, a routing mechanism is proposed with the provision of a handling failover scenario. This technique reduces the energy consumption overhead imposed due to fully dynamic clustering and the $\mathrm{CH}$ selection process. The main contributions of this work are as follows:

1) An energy-efficient unified clustering and routing solution with unequal clustering in Wireless Sensor Networks (WSNs). The solution is based on the position of the nodes in the area and the distance from the BS. For every fixed-area cluster, the $\mathrm{CH}$ is selected based on the cumulative weights of the residual battery, the centrality and the connectivity of the node.

2) A routing solution that handles fail-over scenarios by providing alternative routing paths to any selected $\mathrm{CH}$.

3) The proposed solution is for large-scale WSNs and is suitable for multi-hop communication among devices deployed in a wide area.

The remainder of this paper is organized as follows: Section II elaborates related works in the domain of WSNs; Section III elaborates the proposed Improved Unequal-Clustering and Routing protocol (IUCR) for IoT-based WSNs; Section IV describes the results and analysis and Section V concludes this work.

\section{Related Work}

Unbalanced energy consumption and optimizing network lifetime considered as major research problems in different recent studies [5]-[7], [10]-[14]. For solving a hot-spot problem, a virtual force (gravitation and repulsion) is proposed in [11]. This algorithm distributes nodes uniformly using these forces. The BS is positioned at the center and multiple equalwidth circular regions (annuluses) are formed around it. The concept of virtual gravitational force is used to position the nodes inside each annulus. The routing of nodes is enabled through a "data forwarding area," where optimal paths are selected for balanced energy consumption of nodes.

$\mathrm{CH}$ is selected based on generic utility function that represent degree of satisfaction in relation to consumed transmission power [25]. The utility function is formulated as a noncooperative game and its unique Nash equilibrium point is determined, in terms of devices optimal transmission powers. The proposed approach enables the devices of a cluster with the support of the cluster-head to harvest and store energy in a stable manner through Radio Frequency (RF) signals adopting the wireless powered communication (WPC) paradigm, thus prolonging the operation of the overall M2M network. Each Machine to Machine (M2M) device is associated with a generic utility function, which appropriately represents its degree of satisfaction in relation to the consumed transmission power. The overall combined communication interest among the M2M devices is determined based on three factors, i.e., interest of interaction among the M2M devices regarding the examined IoT application, physical proximity and energy availability.

Node clustering is achieved by combined K-medoids that is a machine learning algorithm with affinity propagation method [26]. K-medoids technique is utilized to find number of clusters and the optimal center of clusters. Modified K-medoids is further utilized to for the topology of the network by iteration. This method is better because traditional K-medoids method applies homogenous clustering and convergence rate.

A clustering scheme based on k-mean clustering method and selection of cluster head through fuzzy logic system is provided in [31] that use genetic algorithm. Similarly, in another work [32], the selection of optimal $\mathrm{CH}$ is performed by firefly technique with cyclic randomization. The $\mathrm{CH}$ is selected in order to make it closer to both base station and nodes. This can help in reducing the time delay. A routing scheme based on $\mathrm{CH}$ is proposed in [33]. The parameter used were the distance variance, dual $\mathrm{CH}$ division and node democracy. It uses the priority function for $\mathrm{CH}$ based on node position and remaining energy.

Another work considered adjustable transmission range and node relocation to repair the hot-spots created during the data transmission process [12]. The algorithm uses cluster overlapping area and the residual energy of nodes to select a suitable node to relocate at coverage hole. A similar node relocation technique is adopted to heal the coverage hole using the multi-objective particle swarm optimization (MOPSO) algorithm based on an adaptive grid [13]. The mobile nodes are rechargeable and moved to optimal locations to meet full target coverage. Subsets in clusters are proposed to jointly handle the data transmission schedule, which helps to extend the network lifetime [27].

$\mathrm{CH}$-selection-messages overhead is considered and reduced using Powell's conjugate gradient method in [14]. This work further uses mobile sinks for data collection to increase network lifetime.

Fixed-area-based equal sized clusters are proposed in [24] for network lifetime improvement. The authors of the work propose deciding the cluster area and cluster members $(\mathrm{CMs})$ before the data transmission operation starts. Unlike a dynamic clustering protocol, which changes their cluster size and number of CMs in every iteration, [24] suggests changing only the $\mathrm{CH}$ based on the residual battery and the node degree (number of connected neighbors of the node). The idea is promising but needs improvement in cluster size optimization for clusters near the BS due to the dual responsibilities of data collection from their own cluster members and data forwarding of other $\mathrm{CHs}$ and $\mathrm{BS}$.

Energy Efficient and Coverage-Guaranteed Unequal-Sized Clustering (ECUC) [17] is another scheme based on adjusting the size of clusters for optimizing network performance in terms of network lifetime. ECUC protocol uses unequal clustering with the BS positioned at center of the circular region where the whole circular region is divided into equalsized circular sectors (also called corona); each sector is 
further divided into an equal number of clusters. The major drawback of this approach is the selection of an equal number of clusters in every corona ignoring the requirement. However, ECUC provided better performance as compared with other protocols such as Distance-Based Segmentation (DBS) [21] and Optimal Clustering in Circular Networks (OCCN) [22]. OCCN is based on same-size clusters with the assumption that global positioning system (GPS) is configured on nodes and the BS is location-aware and positioned at the center of the circular region.

The idea of dividing the $\mathrm{CH}$ load for data collection and relaying is proposed in Energy-Efficient LifeTime Maximization (EELTM) approach [23], which utilizes the intelligent techniques Particle Swarm Optimization (PSO) and Fuzzy Inference System (FIS) [23]. This technique determines the optimal $\mathrm{CH}$ and relay nodes using fitness values determined by the PSO technique. The selected $\mathrm{CH}$ only collects data from its member nodes while the relay node is responsible for forwarding this data to the BS. However, variable size clusters are used in the technique without discussing their impact on the overall network lifetime.

A simplified clustering and $\mathrm{CH}$ selection process based on dynamic cluster radius and intersection region node division scheme is proposed in Energy-balanced clustering routing (EBCR) [18]. The model is optimized by analyzing cooperation and competition among cluster heads. The inter-cluster cooperative routing algorithm greatly improves the transmission efficiency between cluster heads.

A clustering and routing protocol based on the traditional cluster structure and hierarchical routing model using a Markov game is proposed in [19]. The core idea is to control the loop distance of each layer to adjust the relationship between the amount of transmitted data, the transmit power and the number of nodes. The average energy consumption of the nodes in each layer is equal, so that the electrical energy loss between the layers reaches an equilibrium, thereby weakening the hot-spot effect and increasing the life of the network. At the same time, in the inner layer of the same level, in order to balance energy consumption, the Markov game model is introduced, and the gain of the node is set to be related to the residual energy of the node, and the possibility of state transition of the sensing node is calculated.

Partitioning of clusters into zones and assigning $\mathrm{CH}$ responsibility to the node having highest remaining energy and the largest number of neighbors is proposed in [20]. Besides, the zones of each cluster are divided into sub-zones to reduce the energy consumption of the nodes located far from the $\mathrm{CH}$. The proposed protocol also supports multi-hop routing to transmit data from nodes farther from the BS.

All these approaches address the hot-spot problem. In the following section, unequal and fixed-area clusters are proposed to increase network lifetime.

\section{Improved UneQUAL-CLUSTERING AND ROUTING PROTOCOL}

The protocol presents an idea of clustering where CMs are restricted to a defined clustering area. The network area is

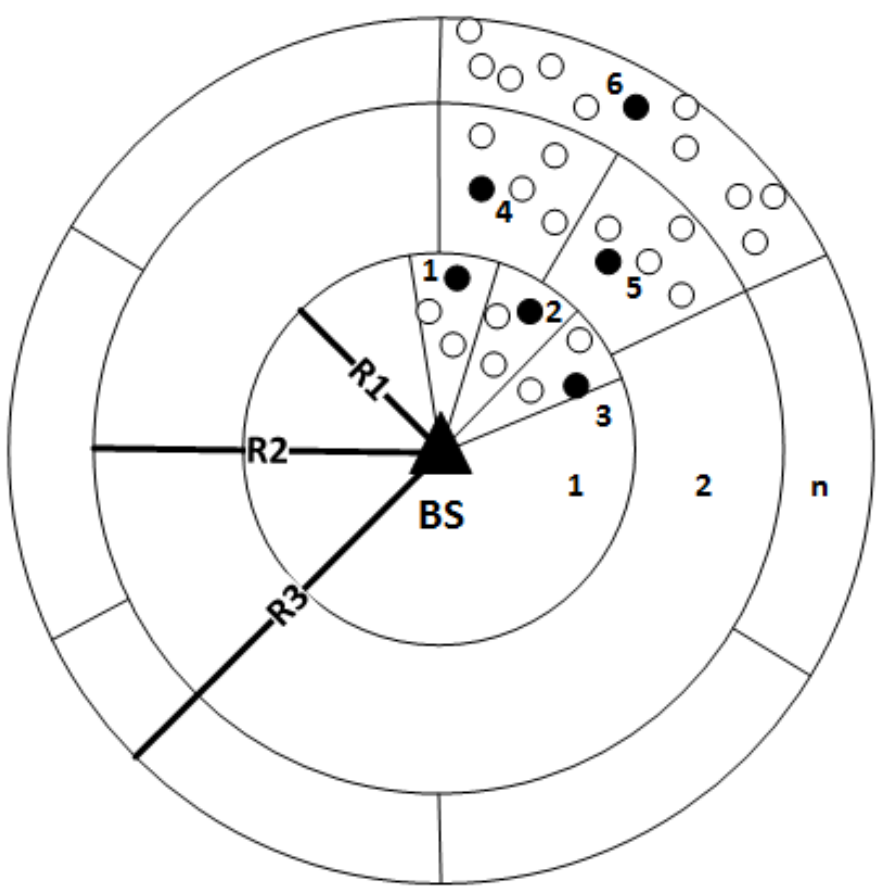

Fig. 1. Unequal Clustering

divided in varying size circular regions, each called Corona (Figure 1). The width of coronas decreases when moving away from the BS. Each corona contains different number of clusters. These fixed area clusters are calculated in such a way that nodes closer to the BS form small area clusters, i.e., the cluster size increases as the distance from the BS increases. The clustering of nodes is performed based on a fixed area that is defined using the transmission range of the node. However, modern IoT devices have transmitters with adjustable transmission range. A first-order radio model is used in the protocol for calculating energy consumption during the transmission and reception of data [24]. All variables and notations used in the proposed protocol are provided in Table I. The algorithm adopted in the protocol is represented as flowchart in Figure 2.

First order radio model is considered during simulation process. In first-order radio model [28]-[30], energy spent for k-bit data transmission is given by:

$$
E_{t x}(\mathrm{~K}, \mathrm{~d})=\left(E_{t x}+E_{d} * d^{n}\right) * K
$$

where $E_{t x}$ is the energy dissipated in the transmission circuit for a single bit, $E_{d}$ is the single bit amplification energy, $E_{d} *$ $d^{n}$ is the energy dissipated for a single bit transmission over a distance $\mathrm{d}$, and $\mathrm{n}$ is the path loss exponent. The value of $\mathrm{n}$ is 2 for free space and 4 for multi-path space. Thus, the total energy dissipated for transmission of the k-bit packet is $E_{t x}(K, d)$.

Similarly, if $E_{r x}$ is the energy required to receive a single bit, then the energy required to receive the K-bit packet is

$$
E_{r x}(\mathrm{~K})=E_{r x} * K
$$

The process is initiated when BS broadcast the position information in overall network and the nodes in the network 
receive this message. First circular corona is created by BS based on minimum transmission range of nodes. Subsequently, variable thickness coronas are created using equation 6 . Radius of each corona is calculated 6 . The clustering process starts from outermost corona using equation 7 . This process defines fixed clustering area. Before data transmission phase, each node sets a backoff timer based on accumulative weight computed using equation 16. The node with highest accumulative weight will set the least timer value. As soon as, the timer of a node expires, it broadcasts an Advertisement (ADV) containing the node ID, corona ID and Area ID. On receiving other nodes in the cluster, the stop their timer and send ACK message with Node-ID, CH-ID, Corona ID and Area ID. Once ACK received from all nodes, $\mathrm{CH}$ broadcasts data transmission TDMA schedule among node. In next step, the $\mathrm{CH}$ sets its forward node (another $\mathrm{CH}$ in inside Corona) for data transmission (the details are given in Routing section (IIIC). Data is collected and transmitted to next $\mathrm{CH}$ in a round. In next round, the $\mathrm{CH}$ is updated again and topology is updated as well.

TABLE I

VARIABLES AND DEFINITIONS FOR IUCR PROTOCOL

\begin{tabular}{ll}
\hline Variable & Definitions \\
\hline$R_{n}$ & The radius of corona $n$ \\
$R_{1}$ & The radius of the innermost circular area \\
$T r_{\min }$ & The minimum transmission range \\
$T r_{d}$ & The data transmission range \\
$\alpha$ & Computed factor between two corona \\
$R_{\max }$ & The maximum radius \\
$N C_{n}$ & The number of clusters \\
$A C_{n}$ & The area of a corona $n$ \\
$A_{n}$ & The area of the outer corona \\
$W_{c}$ & Combined weight of candidate $\mathrm{CH}$ \\
$W_{m}$ & The maximum accumulative weight \\
$\beta$ & The weight of residual battery \\
$\gamma$ & The weight of node degree \\
$\delta$ & Centrality weight \\
$B$ & The residual battery of node \\
$C$ & The centrality value of cluster \\
$D$ & The degree of node \\
$T$ & Back-off timer of a node \\
$F(x)$ & forward nodes of the node $(x)$ \\
$B(x)$ & Backward nodes of the node $(x)$ \\
$N_{F}$ & Number of forward nodes \\
$N_{B}$ & Number of backward nodes \\
&
\end{tabular}

\section{A. Setup Phase}

First phase is setup phase. In equal area-sized clustering, the nodes close to the BS expires earlier than farther nodes. Therefore, we propose an unequal-sized clustering solution, where the BS is positioned at the center of the overall network area, and larger numbers of clusters are formed closer to the $\mathrm{BS}$ and reduce as one moves away from the BS (Fig. 1).

For formation of the clustering area, we make following assumptions:

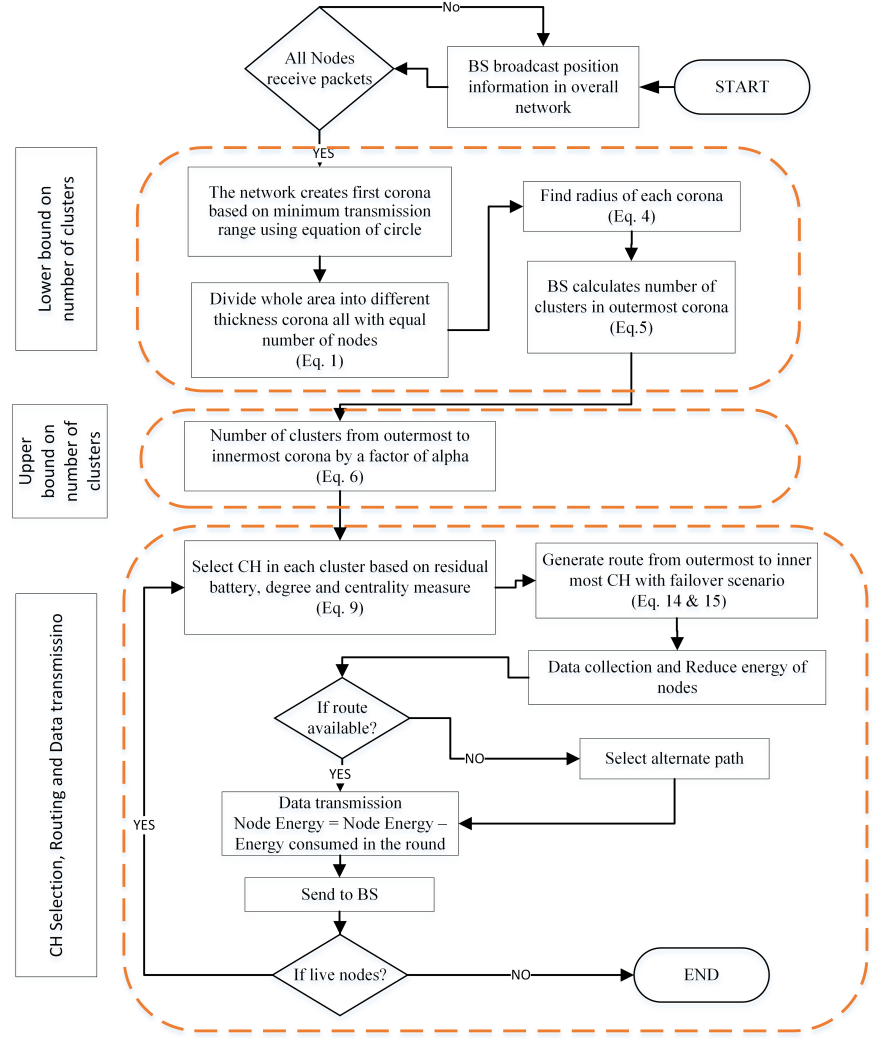

Fig. 2. The flowchart of the IUCR scheme phases

- Nodes are uniformly distributed and randomly located inside the network area.

- Each circular region is called a corona. The number of nodes in each corona is the same. The number of nodes in each cluster in a corona is the same.

- $R_{n}$ is the radius of corona $n$.

- $R_{1}$ is the radius of the innermost circular area where $R_{1}<T r_{\text {min }}$, and $T r_{\text {min }}$ is the minimum transmission range.

The area of each corona is conditioned such that

$$
\pi\left(R_{n}\right)^{2}-\pi\left(R_{n-1}\right)^{2}=\pi\left(R_{n-1}\right)^{2}
$$

Solving for $R_{n}$,

$$
\begin{gathered}
\left(R_{n}\right)^{2}=\left(R_{n-1}\right)^{2}+\left(R_{n-1}\right)^{2} \\
\left(R_{n}\right)^{2}=2\left(R_{n-1}\right)^{2} \\
R_{n}=R_{n-1} \cdot \alpha
\end{gathered}
$$

where $\alpha=\sqrt{2}$

Thus, knowing the maximum radius, $R_{\max }$ of the corona, the BS calculates area of every corona. The next step is unequal clustering. For this, knowing the $R_{\max }$ of the outermost sector, the number of clusters, $N C_{n}$ can be calculated as:

$$
N C_{n}=\frac{2 \pi R_{\max }}{T r_{\min }}
$$


This $N C_{n}$ also serves as the lower bound on the number of clusters and represents the minimum number of clusters in the outermost (nth) corona. Moving from outwards to inside towards the BS, the number of clusters can be calculated as:

$$
N C_{n-1}=N C_{n} \cdot \alpha
$$

Thus, the innermost corona have the highest number of clusters (upper-bound). The area of a corona $n, A C_{n}$, can be calculated as:

$$
A C_{n}=A_{n}-A_{n-1}
$$

where $A_{n}$ is the area of the outer corona and $n>1$. Given the radii of the outer corona, $A_{n}$ and the area of inner corona, $A_{n-1}$, the area of a corona $n$ can be calculated as follows:

$$
A C_{n}=\pi\left(R_{n}^{2}-R_{n-1}^{2}\right)
$$

where, $R_{n}$ is the radius of the outer corona and $R_{n-1}$ is the radius of the inner corona.

1) $\mathrm{CH}$ Probabilities: According to IUCR protocol, the $\mathrm{CH}$ will be selected based on three conditions when its timer expires. These are as follows:

- The accumulative weight of node $(x)$ is greater than the weight of all other candidate nodes in the cluster.

- node $(x)$ must have at least one forward node $(u)$, which also acts as the backward node of node $(x)$. For node $(u)$ to be selected as the forward node of node $(x)$, the probability is

$$
\prod_{u}[1-P(x, u)] \cap u \in F(x)
$$

where $F(x)$ are the forward nodes of node $(x)$. When $\operatorname{node}(x)$ is the only $\mathrm{CH}$ that forwards data to node $(u)$, the probability can be written as follows:

$$
P(x)=\left[1-\prod_{u}(1-P(x, u))\right] \cap u \in F(x), A(x) \neq A(u)
$$

where, $P(x, u)=P_{C H}(u) \cdot P(F(x))$.

- If there is no backward node for node $(x)$, it means that the corona of $\operatorname{node}(x)$ is the last corona. The equation of $\operatorname{node}(x)$ that is to be $\mathrm{CH}$ can then be written as follows:

$$
P_{C H}(x)=P(x, m) \cap N_{B}(x)=0 \cap N_{F}(x) \neq 0
$$

where, $P(x, m)=P_{C H}(m) \cdot P(E Q U A L(x))$ and $m$ is the equal node of node $(x)$ within the same corona but a different cluster.

The number of forward nodes of node $(x)$ can be determined by knowing the data transmission range, $T r_{d}$ of node. Let $n$ be the current corona and $n-1$ is the next corona having forwards nodes, $u_{i}$. The number of forward nodes $N_{F}(x)$ of the $\operatorname{node}(x)$ can be determined as follows:

$$
N_{F}(x)=d\left(x, u_{i}\right)<T r_{d} \cap \forall u_{i} \in A(n-1) \cap i=(1,2, \ldots n)
$$

TABLE II

Network Parameters

\begin{tabular}{ccc}
\hline Parameter & Value & Units \\
\hline Number of nodes & 100,300 & \\
Initial energy & 1.0 & Joules \\
Area of Network & $\mathrm{R}=50-200$ & meters \\
Min Transmission range $\operatorname{Tr}_{\text {min }}$ & 50 & meters \\
Packet size & 2000 & $\mathrm{Bits}$ \\
Control packet length & 200 & $\mathrm{Bits}$ \\
Transmitter energy $T_{x}$ & 50 & $\mathrm{~nJ} / \mathrm{bit} / \mathrm{m}^{2}$ \\
Receiver energy $R_{x}$ & 50 & $\mathrm{~nJ} / \mathrm{bit}^{2} / \mathrm{m}^{2}$ \\
Data aggregation energy & 5 & $\mathrm{~nJ} / \mathrm{bit} / \mathrm{m}^{2}$ \\
Transmit amplifier (free space) & 100 & $\mathrm{~nJ}$ \\
Transmit amplifier (multipath) & 0.0013 & $\mathrm{~nJ}$ \\
\hline
\end{tabular}

where $d(x, u)$ is the distance between node $x$ and $u$, where $i$ is the number of nodes in the corona.

Similarly, the number of backward nodes $N_{B}$ of corona $A(n)$ can be calculated if the current corona is $n$ and the next corona away from the BS is $n+1$. The number of backwards nodes $N_{B}(x)$ for node $x$ can be calculated as follows:

$$
N_{B}(x)=d\left(x, v_{i}\right)<T r_{d} \cap \forall v_{i} \in A(n+1) \cap i=(1,2, \ldots n)
$$

where $d(x, v)$ is the distance between node $x$ and $v$.

2) Cluster-Head Selection: CHs will be selected based on the accumulative weights of three different but related parameters of nodes. These parameters are as follows:

- Residual Battery: The residual battery of a node. The higher the residual battery values, the higher will be the weight of this factor.

- Node Degree: How many nodes are in the range of the node outside the cluster boundary. This parameter ensures the availability of the next hop neighbor for fail-over proof routing [24]. Higher degrees have higher weights and vice versa.

- Node Centrality: Nodes in clusters are positioned at random positions. However, the locations of these nodes are already known by the BS. Based on the position of nodes, the central position of the nodes can be calculated by averaging the $x$ and $y$ coordinates of all the nodes in the cluster. The nodes close to this centroid have a high centrality value, $C$ while nodes away from the centroid have a low centrality value. For simplicity, the closest node has the value 1 and the farthest node has the value 0 .

Selection of a $\mathrm{CH}$ close to the central position in the cluster reduces the overall distance that the member nodes have to send data to the BS and therefore, also reduces the data transmission energy.

The equation for weight is as follows:

$$
W_{c}=\beta \cdot\left(\frac{B_{\max }-B}{B_{\max }}\right)+\gamma \cdot\left(\frac{D_{\max }-D}{D_{\max }}\right)+\delta . C
$$

where, $\beta+\gamma+\delta=1, \beta$ is the weight of the residual battery, 
$\gamma$ is the weight of node degree and $\delta$ is centrality weight based on the distance from the center of the nodes in the cluster. $B$ is the residual battery of the node, $C$ is the centrality value and $D$ is the degree of node, which decreases after every data transmission round due to the connected nodes dying out.

Let $W_{m}$ be the maximum accumulative weight any node can have. This can be defined as follows:

$$
W_{m}=\left(\beta \cdot B_{\max }+\gamma \cdot D_{\max }+\delta . C\right)
$$

where $B_{\max }$ is the maximum battery of a node, $D_{\max }$ is the maximum degree a node and $C$ is the centrality value of a node.

$T$ is the time set for every node in a cluster with the value $\left(W_{c}\right)$. The node with highest value of residual battery, degree and centrality will get its timer, $T$, zero first and sends an advertisement message (ADV) in the minimum transmission range, $T r_{\text {min }}$. This ADV message contains ( $\mathrm{CH}-\mathrm{ID}$, corona ID, Area ID). On receiving the message, all candidate nodes will stop their timer and send back an ACK message with (Node ID, CH-ID, corona ID, Area ID). On receiving the ACK message from cluster members, the $\mathrm{CH}$ broadcasts a TDMA schedule containing the node IDs and their time slot. On receiving this message, the CMs sleep until its time slot.

\section{B. Network Topology Generation}

The development of network topology is initiated by the BS. The traffic flow direction is implicitly defined by maintaining the number of hop counts towards the BS from the node. In the routing table at each node, only the forward nodes towards the BS are selected. A simple topology generated by the BS for the network is shown in Figure 1.

In the first step, one-hop neighbor is initiated by the BS. This includes $\mathrm{CH}$ nodes 1,2 and 3. Each $\mathrm{CH}$ maintains a routing table indicating its forward and backward nodes. The cumulative routing table is given in Table III.

In the next phase, each node in Corona (2) sends a route update message to BS. The nodes in Corona (2) select nodes $(1,2$ and 3$)$ as their forward nodes and node 6 as their backward node (Table IV).

Similarly, node 6 in Corona (n) select as their forward nodes nodes 4 and 5 . Each node maintains its routing table for forward and backward nodes. It sends an update message in case of a change in the $\mathrm{CH}$ in any corona (Table $\mathrm{V}$ ).

After $\mathrm{CH}$ selection and network topology generation, route discovery and selection are performed in the third step. The objective of this step is to provide an optimized path for data transmission from source to sink considering load balancing, fail-over and energy-efficient scenarios. During selection, the path with the least energy consumption is preferred. From source to the $\mathrm{BS}$, intermediate $\mathrm{CHs}$ act as relay nodes. We assume relaying energy as zero to achieve accurate and general results that consider all different types of nodes. This assumption can later be relaxed because relaying only requires the forwarding of packets from one node to the next node based on the routing table.

\section{Routing}

The scenario of routing is described in Figure 1. Initially, BS broadcast route update message to all clusters in Corona 1. On receiving the messages, all the nodes in Corona 1 set the BS as its forward node and broadcast the message in their data transmission range $T r_{\min }$. The node in Corona 2, on receiving the messages from the nodes in Corona 1 , set these nodes as their forward nodes. Corona 1 nodes send back the ACK message in $T r_{\text {min }}$ and set the nodes in Corona 2 as their backward nodes. This process is continued until all the nodes in the different areas have set their forward and backward nodes. Corona 1, 2 and $n$ are shown in Figure 1 where Corona 1 is shown with three clusters, Corona 2 with 2 clusters and Corona $n$ with one cluster.

TABLE III

INITIALIZATION OF NETWORK TOPOLOGY: STEP 1

\begin{tabular}{ccccc}
\hline ID & Forward & Backward & Equal & Hop-count \\
\hline 1 & BS & 4,5 & 2,3 & 1 \\
2 & BS & 4,5 & 1,3 & 1 \\
3 & BS & 4,5 & 1,2 & 1 \\
\hline
\end{tabular}

TABLE IV

INITIALIZATION OF THE NetWork TOPOLOGY: Step 2

\begin{tabular}{ccccc}
\hline ID & Forward & Backward & Equal & Hop-count \\
\hline 4 & 1,2 & 6 & 5 & 2 \\
5 & 2,3 & 6 & 4 & 2 \\
\hline
\end{tabular}

TABLE V

INITIALIZATION OF THE NETWORK TOPOLOGY: STEP 3

\begin{tabular}{ccccc}
\hline ID & Forward & Backward & Equal & Hop-count \\
\hline 6 & 4,5 & Nil & Nil & 3 \\
\hline
\end{tabular}

\section{Results}

To evaluate the performance of IUCR scheme, numerical simulation experiments are carried out in Matlab (Table II). The performance of the protocol is evaluated in terms of network lifetime and total energy consumption. For the experiment, the weight of the residual battery $(\beta)$, node degree $(\gamma)$ and node centrality $(\delta)$ are set at $0.5,0.3$ and 0.2 , respectively. However, these weights are flexible and can be tested for different network topologies. Furthermore, ECUC [17], EELTM [23] and OCCN [22] are considered as benchmarks against the proposed IUCR protocol. All of these protocols are state-ofthe-art works that have been published in last three years in key journals in the domain. These protocols consider unequal clustering with deployment of nodes in a circular area and BS positioned at the center of the area. ECUC utilizes variable size clustering to optimize the network lifetime, whereas IUCR is based on fixed-area unequal clustering. Both consider circular regions for their network topology. IUCR provides fail-over proof routing by providing alternate routing paths towards the $\mathrm{BS}$, whereas ECUC provides a single routing path during a 


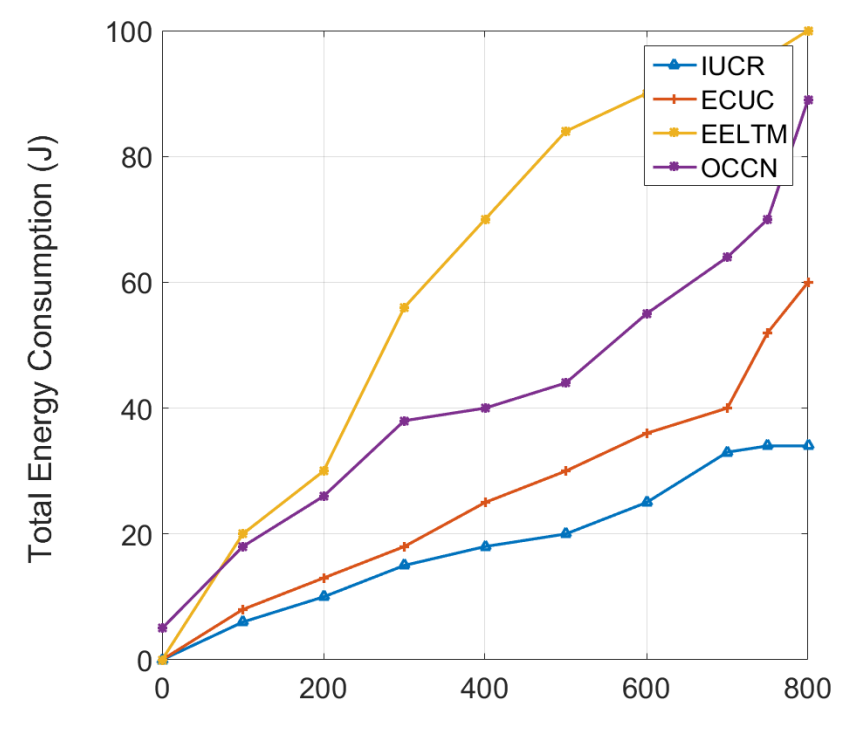

Number of Rounds

Fig. 3. Total energy consumption of network

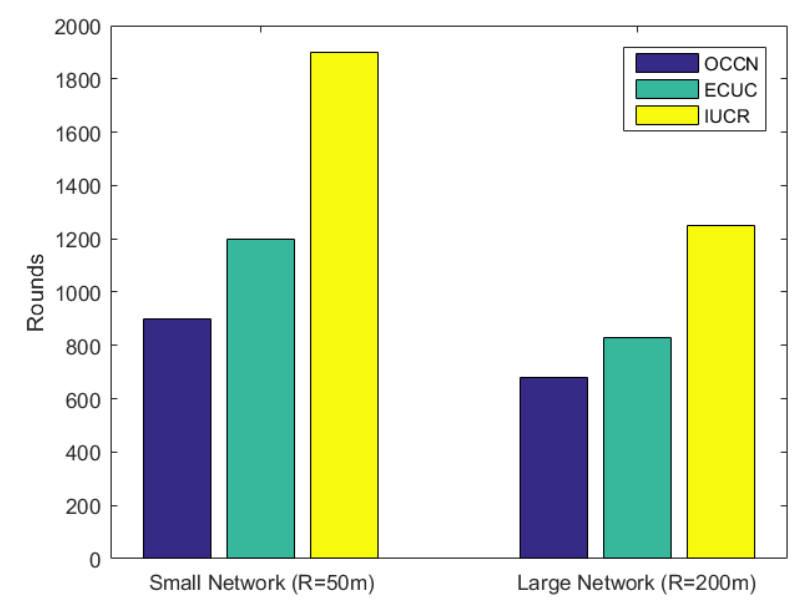

Fig. 4. Network lifetime under two different network area

given round. EELTM [23] proposed increasing lifetime by dividing the $\mathrm{CH}$ load into data collection and data relaying into two nodes. Performance comparison of all protocols is provided in the following sections.

\section{A. Total Network Energy Consumption}

This section discusses the impact on energy consumption on networking with the IUCR protocol. Measurement is taken to show how IUCR reduces the overall network energy consumption, which ultimately results in improved network lifetime. Total energy consumption is defined as the difference between the total initial energy of all nodes in the network and the final energy level of the network that is left in all nodes. A comparison of total energy consumption in network versus rounds in four different protocols is shown in Figure
3. IUCR reduces the overall energy consumption by $50 \%$ as compared to ECUC [17], 33\% as compared with OCCN [22] and $28 \%$ as compared with EELTM [23]. This is because in the ECUC scheme,the same pattern of clusters is formed due to equal clustering. This increases the routing overhead on the nodes closer to the BS. EELTM [23] is based on variable size clusters with different responsibilities of data collection and data forwarding nodes in the cluster. IUCR distributes the data forwarding responsibility more to the nodes closer to the BS. This responsibility gradually distributes into forwarding and collection as one moves away from the BS. The innermost clusters are formed with a data-forwarding centric approach, where each cluster has a few cluster members and a $\mathrm{CH}$ that acts primarily as the data forwarder. The outermost cluster has a larger number of nodes, where the BS serves as the data collector. Equal cluster size results in an imbalance in the energy consumption during inter-cluster communications and can cause an increase in the energy consumption of the network.

\section{B. Network Lifetime}

In this section, a simulation experiment has been done to evaluate the network lifetime. The network lifetime is defined as the time when the first $\mathrm{CH}$ exhausts its battery. Figure 4 shows the plotted network lifetime in terms of rounds in two different schemes under two different scenarios. The simulation results show that the IUCR scheme outperforms ECUC as it has a longer network lifetime by $58 \%$ in small networks and $33 \%$ in large networks. Similarly, IUCR has $110 \%$ longer network lifetime for small networks and $80 \%$ longer network lifetime for larger networks as compared with OCCN [22].

In addition, in cluster-based schemes, a significant amount of energy is consumed by CHs. Clearly, increasing the number of clusters leads to an increase in the energy consumption of the network. However, IUCR used fixed area clustering principle [24] where only the nodes inside a specific region are considered as candidate nodes and $\mathrm{CH}$ selection process is straightforward. Another major difference between the two protocols is use of energy balance among nodes for data collection and forwarding. IUCR tends to distribute energy equally using unequal clustering and therefore, achieves relatively higher overall network lifetime.

\section{Node Density and Network Lifetime}

Furthermore, the network lifetime increases in all four schemes under small networks. This is because decreasing the size of networks leads to decreased distance between nodes and $\mathrm{CHs}$, resulting in reducing the energy consumption of $\mathrm{CHs}$ and improving the network lifetime. Likewise, Figure 5 shows the lifetime of network of three schemes in low and high density scenarios. As can be seen, the network lifetime increases in all two schemes under low density network scenario. It stems from decreasing the number of member nodes in the low density network, which leads to a decrease in the energy consumption of $\mathrm{CHs}$ and enhances the network performance and lifetime. 


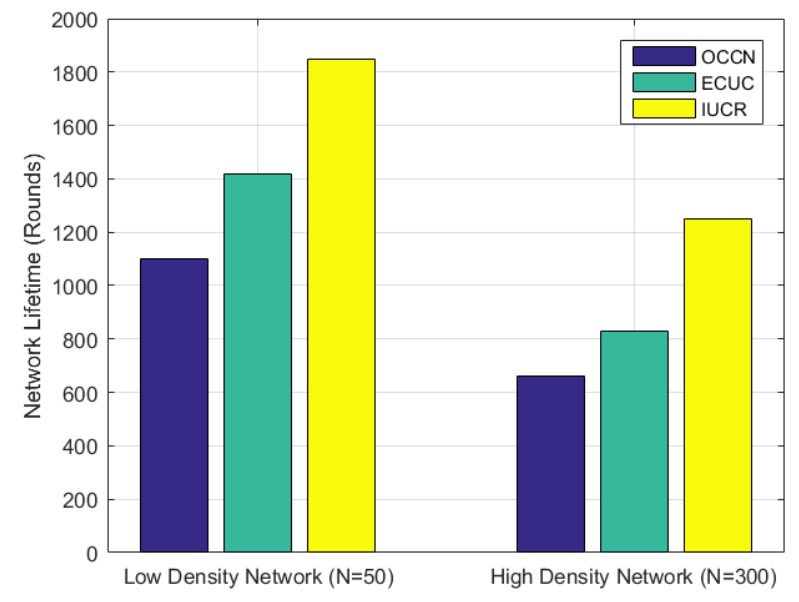

Fig. 5. Network lifetime under different number of nodes

Figure 6 shows the optimal number of clusters in the case of increasing number of nodes. The optimal number increases with an increase in the number of nodes from 100 to 450. This is because ECUC reduces the cluster size and keeps a fixed number of nodes inside a cluster. Increasing the number of nodes increases energy consumption in the cluster. Thus, increasing the number of nodes increases the number of clusters. The number of sectors increases as the number of nodes increases. Consequently, constructing small clusters leads to decreased distances between $\mathrm{CMs}$ and $\mathrm{CHs}$, which results in decreased energy consumption. The IUCR protocol is based on unequal clustering strategy, where BS is positioned at the center of area and clustering areas are formed in circular format. The width of corona varies while maintaining the same number of nodes in all coronas. IUCR divides the circular area based on unequal area size strategy from outwards to inwards in the area to make sure there is balanced energy distribution among the nodes. Therefore, the number of clusters remains the same in the IUCR with an increasing number of nodes.

\section{Energy Consumption Reduction Ratio (ECRR)}

ECRR is the ratio that determines the reduction in energy consumption before and after applying the techniques. The purpose of measuring the ECRR is to show the improvement of ECUC in terms of increasing the influence of clustering on reducing the energy.

$$
E C R R=\frac{\text { EnergyConsumedAfterClustering }}{\text { EnergyConsumedBeforeClustering }}
$$

For ECUC protocols, the number of clusters varies with number of nodes. In this case, ECRR is a measure that provides the impact on energy consumption during the clustering process in the IUCR, ECUC and OCCN protocols. The IUCR protocol is based on fixed-area clusters and the number of nodes decreases with time, while ECUC is based on dynamic clustering, where cluster size varies but tends to keep the number of nodes the same. OCCN performs dynamic unequal clustering. Figure 7 shows a comparison of the ECUC, OCCN

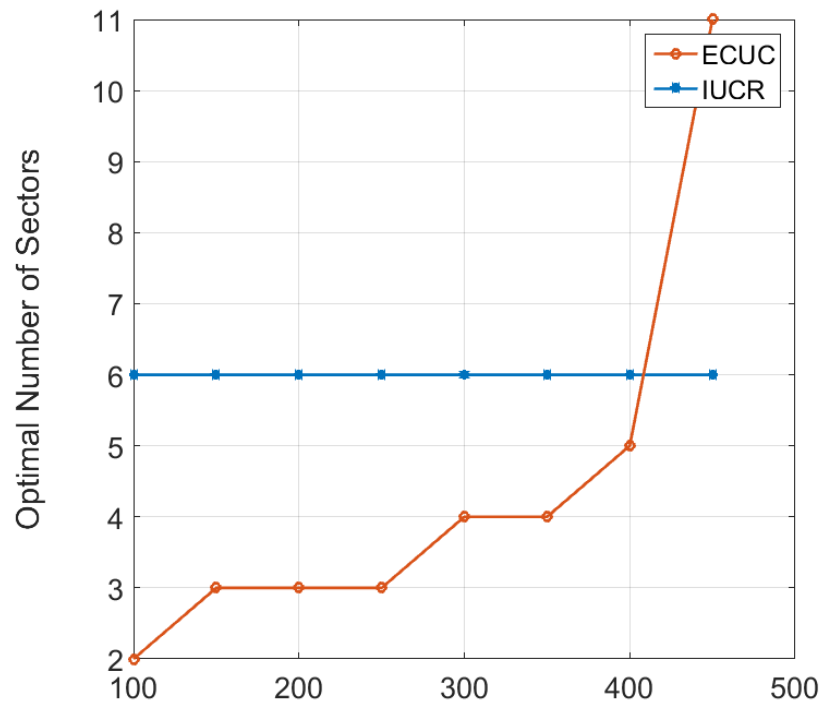

Number of Nodes

Fig. 6. Optimal Number of Clusters with different Number of Nodes

and IUCR protocols in terms of ECRR values in different rounds during the clustering process.

At 200 rounds, $12 \%$ less energy is consumed in the IUCR protocol as compared with the ECUC protocol and $28 \%$ less energy is consumed as compared with OCCN. However, the adoption of dynamic and static clustering strategies in both protocols did not improve ECRR significantly. In ECUC, the energy saved due to the smaller cluster size was exhausted in the process of dynamic clustering itself. Similarly, the energy saved in the IUCR protocol due to fixed-area clustering is consumed on serving larger numbers of CMs. The purpose of unequal clustering mechanisms is to reduce the network energy consumption after applying the clustering process. IUCR significantly improves the energy performance by adopting fixedarea and unequal clustering.

\section{E. Execution Time and Throughput}

The execution time is calculated for circular region of radius 100 meter, 200 meters and 400 meters. The initial energy of nodes is selected to be 0.25 joules, 0.5 Joules and 1.0 Joules. It can be seen from the results (tables VI,VII,VIII) that the execution time for different network sizes is same but number of packets reaching to BS reduces with increase in area size. The reason is the depletion of energy of intermediate nodes and finding alternative paths to reach the BS. Also increasing the size of network increase number of hop counts to BS.

For given radius of 100 meter, number of packets reaching to BS is shown in the table (IX) for different values of initial energy of nodes. Energy consumed for sending the data from $\mathrm{CH}$ to BS is also provided. It can be seen that very less energy is consumed for data transmission and most of the energy is utilized for management activity of the clustering and routing. 


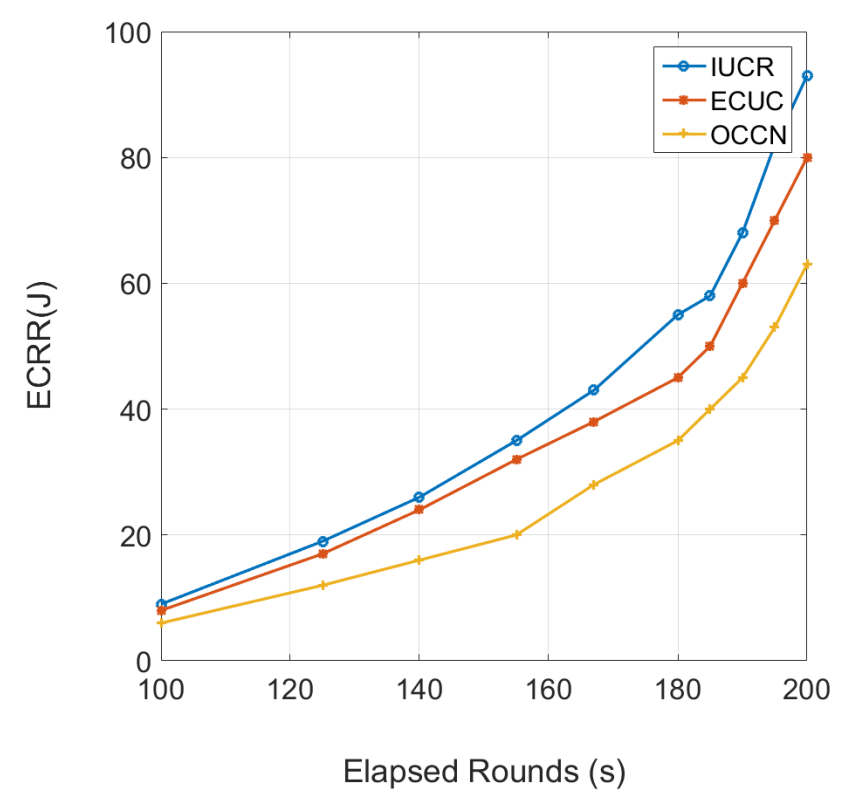

Fig. 7. Energy Consumption Reduction Ratio.

TABLE VI

EXECUTION TIME (RADIUS $=100$ METERS)

\begin{tabular}{cccc}
\hline Init Energy & Execution Time (seconds) & Rounds & Packets \\
\hline 0.25 & 190 & 1874 & 36326 \\
0.5 & 390 & 3651 & 70801 \\
1.0 & 660 & 6451 & 144730 \\
\hline
\end{tabular}

\section{F. Routing Performance}

The IUCR protocol addresses clustering and routing as interlinked issues. The performance of routing in the IUCR protocol is compared with the O-LEACH [28], DL-LEACH [29] and BPA-CRP [30] protocols. The number of connected nodes, network lifetime and energy consumption performance are used as evaluation parameters. The radius of area is 50 meters and 0.5 Joules as the initial energy of all nodes.

The number of clusters formed is based on the transmission range of the nodes. For smaller transmission ranges, although JCR performs better than IUCR, the results are comparable. However, for a given radius of $50 \mathrm{~m}$, IUCR is flexible in creating clusters according to the transmission range, while JCR fails to do that and still creates more clusters (Fig. 8). JCR does not adjust the number of clusters when $T r_{\text {min }}$ is more than $30 \mathrm{~m}$, while IUCR succeeds in efficiently adjusting to larger transmission ranges.

The average hop count to the sink node is another measure. We compare the average hop count to sink between JCR and IUCR. As the transmission range of nodes increases from $20 \mathrm{~m}$ to $50 \mathrm{~m}$ with radius of area is 50 meters, the average hop count of both protocols demonstrates comparable variation (Fig. 9). When $T_{r}$ ranges from $20 \mathrm{~m}$ to $50 \mathrm{~m}$, the average hop count of the CHs increases from 2.64 to 2.76 for the JCR protocol, where it decreases from 2.5 to 1.3 hops. This is because the IUCR protocol is adaptive to variable transmission ranges and

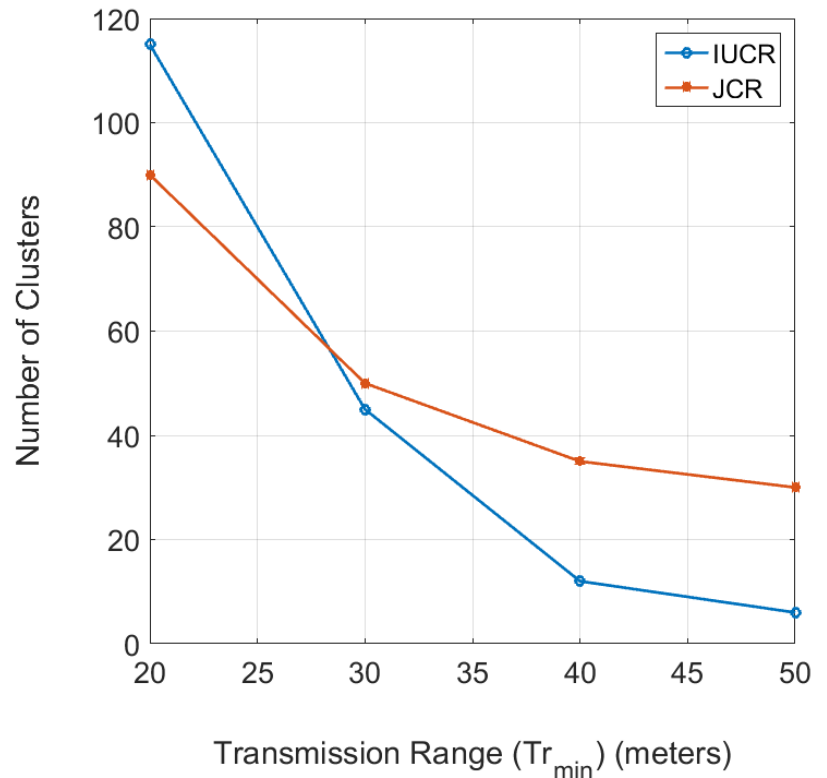

Fig. 8. Transmission range and number of clusters (Radius $=$ 50 meter, $R_{\max }=50$ meter)

TABLE VII

EXECUTION TIME (RADIUS = 200 METERS)

\begin{tabular}{cccc}
\hline Init Energy & Execution Time (seconds) & Rounds & Packets \\
\hline 0.25 & 190 & 1840 & 26295 \\
0.5 & 360 & 3517 & 60897 \\
1.0 & 780 & 7415 & 122702 \\
\hline
\end{tabular}

produces an optimized clustering area. This proves that the transmission path generated by IUCR is more efficient than JCR, and the advantage is greater when $T_{r}$ is larger.

The number of connected nodes is compared in Fig. 10. The number of connected nodes in every round is 100 percent for IUCR, close to 100 percent for O-LEACH and 90-95 percent for BPA-CRP. The reason is that the transmission range of every cluster is fixed, and all nodes within the cluster are covered within the range. However, in O-LEACH, due to gateway nodes, not only the network lifetime decrease but some nodes also remain disconnected. Since O-LEACH is specifically designed for the purpose of minimizing disconnected nodes, it achieves good connected node results.

\section{CONCLUSION}

The proposed protocol presented clustering and routing as unified problems for circular area networks. The protocol considered the BS at the center of the circular region and cluster-members are distributed around it. Major contributions of this work includes introduction of fixed-area based clusters using an unequal clustering method and uniformly distributing clustering and routing responsibility among all nodes in the network. The technique showed significant performance gain in terms of network life time by $58 \%$ and $110 \%$ respectively on comparing with ECUC and OCCN protocols. Furthermore, 


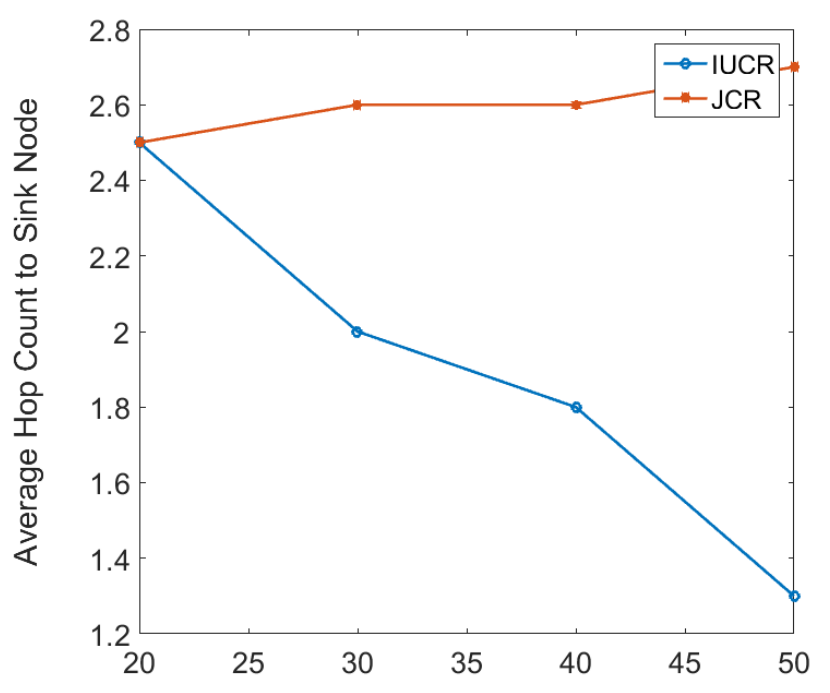

Transmission Range (meters)

Fig. 9. Average hop count to the sink node (Radius $=\mathbf{5 0 m e t e r})$

TABLE VIII

EXECUTION TIME (RADIUS $=400$ METERS)

\begin{tabular}{cccc}
\hline Init Energy & Execution Time (seconds) & Rounds & Packets \\
\hline 0.25 & 120 & 1125 & 11126 \\
0.5 & 470 & 3321 & 22091 \\
1.0 & 925 & 6371 & 44842 \\
\hline
\end{tabular}

the approach exhibits a reduction in energy consumption by $50 \%$ and $33 \%$ as compared with ECUC and OCCN. IUCR protocol considered fixed weights of three different weighing parameters for $\mathrm{CH}$ selection. The work can further be extended by selecting different weights of $\mathrm{CH}$ selection parameters and selecting optimal accumulative weights based on the network area and number of nodes.

\section{REFERENCES}

[1] G. Manogaran, R. Varatharajan, D. Lopez et al., "A new architecture of Internet of Things and big data ecosystem for secured smart healthcare monitoring and alerting system," Future Generation Computer Systems, vol. 82, no. 5, pp. 375-387, 2018.

[2] S. Biswas, R. Das, and P. Chatterjee, "Energy-efficient connected target coverage in multi-hop wireless sensor networks," Industry Interactive Innovations in Science, Engineering and Technology, pp. 411-421: Springer, 2018.

[3] V. Sundararaj, S. Muthukumar, and R. Kumar, "An optimal cluster formation based energy efficient dynamic scheduling hybrid MAC protocol for heavy traffic load in wireless sensor networks," Computers \& Security, vol. 77, pp. 277288, 2018.

[4] O. Bello, and S. Zeadally, "Intelligent device-to-device communication in the internet of things," IEEE Systems Journal, vol. 10, no. 3, pp. 11721182, 2014.

[5] A. AlBaz, and A. ElSayed, "A new algorithm for cluster head selection in LEACH protocol for wireless sensor networks," International Journal of Communication Systems, vol. 31, no. 1, pp. e3407, 2018.

[6] S. K. Singh, P. Kumar, and J. P. Singh, "A survey on successors of LEACH protocol," IEEE Access, vol. 5, pp. 42984328, 2017.

[7] H. Farman, B. Jan, H. Javed et al., "Multi-criteria based zone head selection in Internet of Things based wireless sensor networks," Future Generation Computer Systems, vol. 87, pp. 364371, 2018.

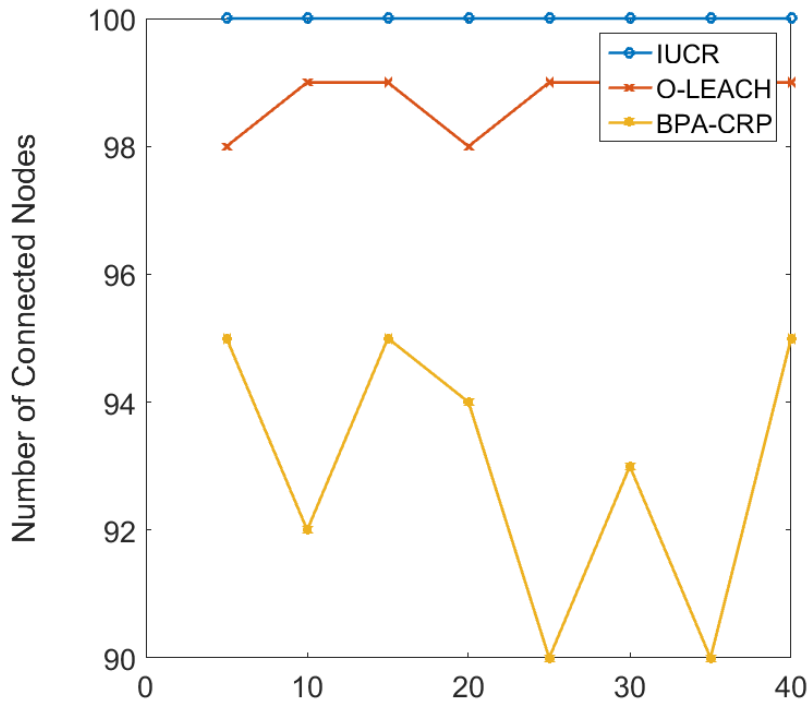

Number of Rounds

Fig. 10. Number of connected nodes (Radius $=\mathbf{5 0 m e t e r})$

TABLE IX

THROUGHPUT AND NETWORK LIFETIME (TRANSMISSION RANGE $=50$

M)

\begin{tabular}{cccc}
\hline Init Energy & Energy Consumed (Joules) & Packets & Rounds \\
\hline 0.25 & 0.00055 & 35638 & 504 \\
0.5 & 0.000123 & 70618 & 998 \\
1.0 & 0.0016275 & 115374 & 1607 \\
\hline
\end{tabular}

[8] W. R. Heinzelman, A. Chandrakasan, and H. Balakrishnan, "Energyefficient communication protocol for wireless microsensor networks." Proceedings of the 33rd Annual Hawaii International Conference on System Sciences. Piscataway, NJ, USA. IEEE, pp. 10, 2000.

[9] W. B. Heinzelman, A. P. Chandrakasan, and H. Balakrishnan, "An application-specific protocol architecture for wireless microsensor networks," IEEE Transactions on wireless communications, vol. 1, no. 4, pp. 660670, 2002.

[10] S. H. Kang, "Energy optimization in cluster-based routing protocols for large-area wireless sensor networks," Symmetry, vol. 11, no. 1, pp. 37, 2019.

[11] C. Sha, C. Ren, R. Malekian et al., "A Type of Virtual Force-Based Energy-Hole Mitigation Strategy for Sensor Networks," IEEE Sensors Journal, vol. 20, no. 2, pp. 1105-1119, 2019.

[12] B. Khalifa, A. M. Khedr, and Z. Al Aghbari, "A coverage maintenance algorithm for mobile WSNs with adjustable sensing range," IEEE Sensors Journal, vol. 20, no. 3, pp. 1582-1591, 2019.

[13] Y. Xiong, G. Chen, M. Lu et al., "A two-phase lifetime-enhancing method for hybrid energy-harvesting wireless sensor network," IEEE Sensors Journal, vol. 20, no. 4, pp. 1934-1946, 2019.

[14] G. Kaur, P. Chanak, and M. Bhattacharya, "Memetic Algorithm-Based Data Gathering Scheme for IoT-Enabled Wireless Sensor Networks," IEEE Sensors Journal, vol. 20, no. 19, pp. 11725-11734, 2020.

[15] Z. Xu, L. Chen, C. Chen et al., "Joint clustering and routing design for reliable and efficient data collection in large-scale wireless sensor networks," IEEE Internet of Things Journal, vol. 3, no. 4, pp. 520-532, 2015.

[16] S. Arjunan, and S. Pothula, "A survey on unequal clustering protocols in wireless sensor networks," Journal of King Saud University-Computer and Information Sciences, vol. 31, no. 3, pp. 304-317, 2019.

[17] N. Gharaei, Y. D. Al-Otaibi, S. A. Butt et al., "Energy-efficient and coverage-guaranteed unequal-sized clustering for wireless sensor networks," IEEE Access, vol. 7, pp. 157883-157891, 2019. 
[18] Y. Yao, W. Chen, J. Guo et al., "Simplified clustering and improved intercluster cooperation approach for wireless sensor network energy balanced routing," EURASIP Journal on Wireless Communications and Networking, vol. 2020, no. 1, pp. 1-33, 2020.

[19] Y. Deng, C. Cao, and S. Chen, "Distributed Energy Equilibrium Routing Algorithm Based on Hierarchical Thought and Markov Game in WSN." Proceedings of the 4th International Conference on Intelligent Information Processing, pp. 368-373, 2019.

[20] F. Khanouche, L. Maouche, F. Mir et al., "Energy efficient Multi-hops Routing protocol based on Clusters Reorganization for wireless sensor networks." Proceedings of the 3rd International Conference on Future Networks and Distributed Systems, pp. 1-10, 2019.

[21] N. Amini, A. Vahdatpour, F. Dabiri et al., "Joint consideration of energyefficiency and coveragepreservation in microsensor networks," Wireless communications and mobile computing, vol. 11, no. 6, pp. 707 722, 2011.

[22] M. Arghavani, M. Esmaeili, M. Esmaeili et al., "Optimal energy aware clustering in circular wireless sensor networks," Ad Hoc Networks, vol. 65, pp. 91-98, 2017.

[23] K. Arikumar, V. Natarajan, and S. C. Satapathy, "EELTM: An Energy Efficient LifeTime Maximization Approach for WSN by PSO and Fuzzy-Based Unequal Clustering," Arabian Journal for Science and Engineering, vol. 45, no. 12, pp. 10245-10260, 2020.

[24] M. A. Alharbi, M. Kolberg, and M. Zeeshan, "Towards improved clustering and routing protocol for wireless sensor networks," EURASIP Journal on Wireless Communications and Networking, vol. 2021, no. 1, pp. 1-31, 2021. https://doi-org.sdl.idm.oclc.org/10.1186/s13638-02101911-9.

[25] E. E. Tsiropoulou, G. Mitsis, and S. Papavassiliou, "Interest-aware energy collection \& resource management in machine to machine communications." Ad Hoc Networks, vol. 68, pp. 48-57, 2018.

[26] J. Wang, Y. Gao, K. Wang et al., "An affinity propagation-based selfadaptive clustering method for wireless sensor networks.", Sensors, vol. 19, no.11, pp. 2579, 2019.

[27] O. B. K. Pentikousis, R. A. Calvo, and S. Papavassiliou, "Mobile networks and management," Springer-Verlag Berlin Heidelberg, 2010.

[28] W. Jerbi, A. Guermazi, and H. Trabelsi, "O-LEACH of routing protocol for wireless sensor networks," in Proc. 13th Int. Conf. Comput. Graph.,Imag. Vis (CGiV), pp. 399-404, Mar. 2016.

[29] J.-Y. Lee, K.-D. Jung, S.-J. Moon, and H.-Y. Jeong, "Improvement on LEACH protocol of a wide-area wireless sensor network," Multimedia Tools Application, vol. 76, no. 19, pp. 19843-19860, Oct. 2017.

[30] K. A. Darabkh, M. Z. El-Yabroudi, and A. H. El-Mousa, "BPA-CRP: A balanced power-aware clustering and routing protocol for wireless sensor networks," Ad Hoc Netw., vol. 82, pp. 155-171, Jan. 2019.

[31] L. Li, and D. Li, "An energy-balanced routing protocol for a wireless sensor network.", Journal of Sensors, vol. 2018, 2018.

[32] A. Sarkar, and T. S. Murugan, "Cluster head selection for energy efficient and delay-less routing in wireless sensor network.", Wireless Networks vol. 25, no.1, pp. 303-320, 2019.

[33] Z. Zhao, K. Xu, G. Hui et al., "An energy-efficient clustering routing protocol for wireless sensor networks based on AGNES with balanced energy consumption optimization.", Sensors, vol. 18, no.11, p3938, 2018 . 